\title{
A Slightly Modified Expression of the Polar Surface Area Applied to an Olfactory Study
}

\author{
Paul Laffort \\ Centre des Sciences du Goût, Dijon, France \\ Email: paul.laffort@u-bourgogne.fr
}

Received August 21, 2013; revised September 20, 2013; accepted September 28, 2013

Copyright (C) 2013 Paul Laffort. This is an open access article distributed under the Creative Commons Attribution License, which permits unrestricted use, distribution, and reproduction in any medium, provided the original work is properly cited.

\begin{abstract}
The polar surface area of a molecule is currently defined as the surface sum over all polar atoms, primarily oxygen and nitrogen, also including their attached hydrogens (named PSA1 in the present study). Some authors also include sulfur and phosphor (PSA3). The slight modification suggested here is based on the fact that it is difficult to consider, on a theoretical point of view, hexavalent $\mathrm{S}$ and pentavalents $\mathrm{N}$ and $\mathrm{P}$ as polar atoms. Indeed, in these cases, all their peripheral electrons are involved in bondings. We propose to define PSA2 using the initial definition extended to O, S, N, P, with the exception of hexavalent $\mathrm{S}$ and pentavalents $\mathrm{N}$ and $\mathrm{P}$. In order to test this hypothesis, the three expressions PSA1, PSA2 and PSA3 have been applied in a QSAR to a physiological phenomenon called comfort olfactory perceived intensity, for the human responses to 186 odorants (QSAR stands for Quantitative Structure Activity Relationship). The PSA2 expression has been selected as the more suitable, associated with two other molecular properties (molar refraction and Van der Waals molecular volume).
\end{abstract}

Keywords: Polar Surface Area; QSAR; Cheminformatics; Olfaction; Honey-Bee; Psychophysics

\section{Introduction}

The polar surface area PSA is a quite fascinating molecular property on various aspects. On one hand, its definition is chemically simple and precise, according to Palm et al. [1]: "the area occupied by nitrogen and oxygen atoms, and hydrogen atoms attached to these heteroatoms". On the other hand, its justification has been considered, in a first time, as strictly pharmacological, in the sense that it mainly reflects the molecular transport properties of drugs, particularly blood-brain barrier (BBB) penetration and intestinal absorption [1,2]. The consideration that PSA could also be involved in the physicochemical field as a general criterion of polarity was unexpected, since, for example, strongly polar elements such as halogens, particularly fluorine, have always been excluded of its definition. We have however recently shown that PSA, associated with the Van der Waals molecular volume $\mathrm{Vw}$, is strongly involved in the characterization of the polarity of gas-liquid chromatography stationary phases [3].

As already pointed out in 2007 by Ertl [4], the number of publications in which PSA is involved has been growing exponentially since 1996. One of the more noteworthy recent ones is due to Muehlbacher et al. [5], who have obtained a discrimination without misclassification between two sets of drugs ( 87 not BBB permeable compounds and 133 BBB permeable compounds), using only four molecular descriptors in which PSA has a key role.

The aim of the present study is firstly to propose, on theoretical considerations, a slightly modified definition of PSA, and then to test if one or another of its expressions reflects, possibly associated with other molecular characteristics, the biological phenomenon summarized hereafter in the particular case of human olfactory responses.

\section{The Comfort Olfactory Perceived Intensity}

This phenomenon can be apprehended in the Figure 1. The top of the figure is a classical representation in log$\log$ coordinates of the olfactory perceived intensity (OLPI) vs. the air concentration for three different odorants. It visualizes an observation firstly shown in 1973 by Laffort and Dravnieks [6] and always verified since: the lower the threshold, the lower the slope, as a general trend with a few exceptions (outliers). It seems that 


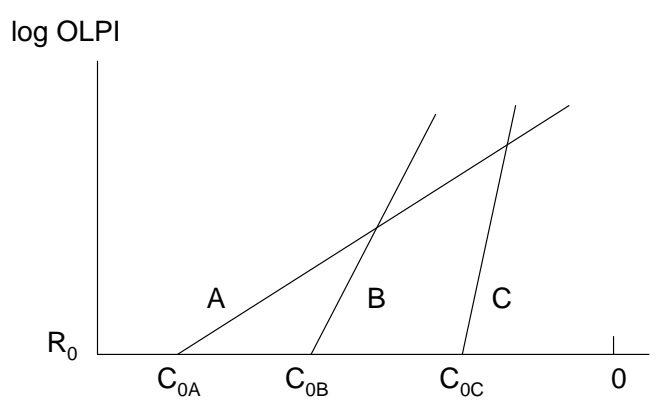

log conc. in air (molar fraction)

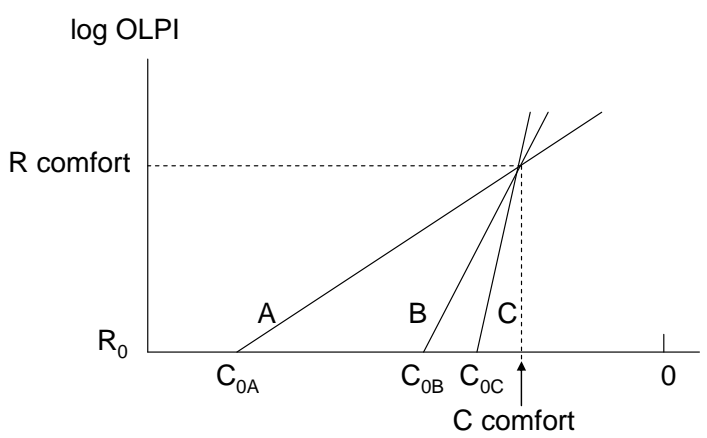

log conc. at the level of receptors

Figure 1. Simplified representation of the comfort model for the olfactory perceived intensity (OLPI), already verified in the honey-bee. Concentrations are expressed in molar fractions. See details in text.

odorant concentration has to be considered at the recaptors level rather than in air, in order to have a complete description of the phenomenon. This transformation is supposed to be obtained using a simple physicochemical property.

The bottom of the Figure 1 visualizes the results we have already verified in the honey bee. Firstly, the convergence of the straight lines using electroantenographical responses vs. fractions of saturated vapor pressures is high $(\mathrm{r}=0.97, \mathrm{~N}=59, \mathrm{~F}=404$, without outliers) [7]. Secondly, the ordinate of this point corresponds to an OLPI value associated with an optimal learning of olfactory recognition using an associating conditioning of the proboscical reflex [8-10]. For this reason we consider the ordinate of this convergence point as reflecting an optimal comfort. The abscissa of the convergence point, identical for all odorants, is the logarithmic value of $1 / 250$ of the maximal possible concentration at the receptor levels (expressed in molar fraction, i.e. -2.4).

Unfortunately, if the saturated vapor pressure at room temperature appears to be a suitable property to transform the top into the bottom of Figure $\mathbf{1}$ for honey-bees, it is not the case for humans [11,12]. The explanation seems to be that the odorants reach the olfactory dendrites via a humid way in vertebrates, and through a dry route into pores-tubules in insects.
It should be noted that a synthesis of the experimentations on honey-bees summarized above has not yet been published except in a shortened form [13], but has been orally presented in Dijon [13] and at the Monell Chemical Senses Center [14].

\section{Methods}

\subsection{Molecular Properties}

Three groups of physicochemical properties have been tested, all derived from the molecular structure.

Properties more or less associated with the molecular size, namely molar mass (M), Van der Waals molecular volume $(\mathrm{Vw})$, Van der Waals molecular surface $(\mathrm{Sw})$, Molar refractivity (MR), molecular polarizability (PIZ), partition coefficient octanol-water (ClogP). All these properties have been got from the Chemaxon calculator-plugins [15]. A seventh expression has been considered, the solvation parameter of dispersion $(\delta)$ according to Laffort and Héricourt [16].

Properties of polar nature, rather independent of the molecular size, namely three different expressions of the polar surface area (PSA).

In a first time, PSA values have been established by Palm et al. $[1,2]$ using sophisticated programs taking into account the molecular three-dimensional shape and its flexibility. Later, a very simple topological method using summation of surface contributions of polar fragments (termed TPSA) has been applied by Ertl et al. [17], exhibiting an excellent correlation with theoretical PSA values $(\mathrm{r}=0.991, \mathrm{~N}=34810$ substances).

The slightly polar atoms sulfur and phosphor have sometimes been taken into consideration $[4,18]$. We have called PSA1 the version with only $\mathrm{O}$ and $\mathrm{N}$ and PSA3 the version with $\mathrm{O}, \mathrm{N}, \mathrm{S}$ and $\mathrm{P}$.

We have added a supplementary version, named PSA2, which includes $\mathrm{O}, \mathrm{S}$ except when it is hexavalent, and $\mathrm{N}$ and $\mathrm{P}$ only when they are trivalent. That implies, among others, the exclusion of $\mathrm{N}$ in nitrates, $\mathrm{S}$ in sulfates, $\mathrm{P}$ in phosphates. This alternative choice is in agreement with the fact, according to the Lewis theory [19], that the peripheral electrons involved in dative (or semi-polar) bonding should totally attributed to the more polar of the two concerned polar atoms. For example, four out five peripheral electrons of $\mathrm{N}$ in organic nitrates should totally be attributed to the two $\mathrm{O}$, whereas the fifth electron is shared in a covalent bonding with one $\mathrm{C}$. Therefore, this type of pentavalent nitrogen should totally be non polar, as it is visualized in Figure 2, and on the contrary to that admitted in the PSA1 and PSA3 versions.

The orientation solvation parameter $\omega$ according to Laffort and Héricourt [16] has been discarded because not predicted with enough accuracy from the $2 \mathrm{D}$ molecular structure. 


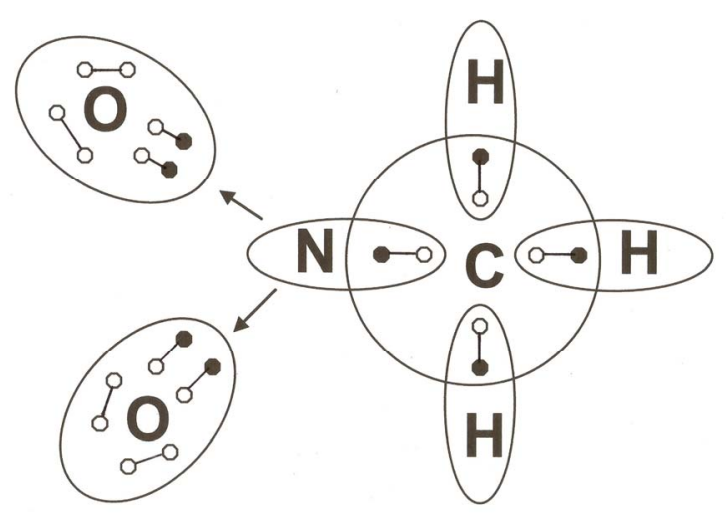

Figure 2. Graphical representation of the four dative (or semi-polar) bonds and the four covalent bonds of nitromethane, according to the Lewis theory [19], clearly showing that the pentavalent nitrogen is not polar (absence of pairs of peripheral electrons not included in the bonding, whereas each oxygen has two pairs).

One induction/polarizability index, also independent of the molecular size. This property is the $\varepsilon$ solvation parameter according to Laffort and Héricourt [16]. It is very similar to the descriptor named R2 or E according to Abraham and co-authors [20,21].

\subsection{Olfactory Properties}

Two experimental olfactory properties are needed to draw the top of the Figure 1 for humans:

- Olfactory thresholds, which are, in some way, the intercept of the straight lines with the $\mathrm{X}$ axis,

- The slopes of the straight lines, currently named power law exponents.

Presently, the most complete and sure compilation of human olfactory thresholds in air has been established in 2011 by Van Gemert [22] and concerns 1150 odorants. However, an issue encountered with this type of compilation is that different authors obtain widely different values for the same substance. We have shown since 1963 [23] that these differences, mainly due to differences in experimental protocols, can be considerably reduced by assigning a specific weighting coefficient to each author's results. Applying this method, Devos et al. [24] have established in 1990 the most recent published collection of standardized thresholds, which concerns 529 odorants. An updated version of this compilation, unpublished, has been established in 1995 for 117 supplementary compounds [25].

It is also well established since 1969 that the exponent values differ, for a given odorant, with the experimental procedure [26]. Applying a similar method as for thresholds, Devos et al. [27] have published in 2002 the most recent and complete collection of standardized human power law exponents in air.
The updated compilation of olfactory thresholds and that of power law exponents according to Devos et al. [25,27], concern in common 194 odorants. Eight compounds have been discarded of this overlapping set:

- Two selenium compounds for which part of the tested molecular properties have not been found (ethanesenelol and diethyl selenide).

- Four charged compounds for which part of the tested molecular properties have not been found (ozone, 2-propenyl isocyanide, phenyl isocyanide and ammonia, i.e. $\mathrm{NH}_{4} \mathrm{OH}$ in aqueous solution).

- Two alcohols presenting very small values of power law exponents (0.11 and 0.08 for 1-nonanol and 1decanol respectively). Indeed, in the explored regressions of $\log$ of thresholds vs. other characteristics, the exponents being expressed in inverse values, they could present an excessive influence in the statistical processing (expressed in inverse values, the $186 \mathrm{kept}$ values represent $1 / 3$ of the total range when these two alcohols are included).

\subsection{Statistical Tools}

In addition to the Microsoft Excel Windows facilities for drawing diagrams and handling data sets, the SYSTAT ${ }^{\circledR}$ 10.2 for Windows has been applied for stepwise MLRA (Multidimensional Linear Regression Analysis).

\section{Results}

\subsection{QSAR (Quantitative Structure/Activity Relationship) for 186 Odorants}

We have compared all together the olfactory properties and the molecular parameters for the 186 compounds described in the Methods section, in order to embrace the larger possible of molecular features. The eleven molecular parameters have been tested in multiple cross combinations using the SYSTAT stepwise MLRA. The best obtained regression is given by Equation (1):

$$
\begin{aligned}
& \text { p.ol }=\frac{0.8177}{n}+\frac{7.406 \times \mathrm{MR}}{100}+3.604 \times \frac{\mathrm{PSA} 2}{\mathrm{Vw}}+2.3 \\
& \text { [66] [142] } \\
& \text { [72] }
\end{aligned}
$$

in which p.ol stands for the negative log of concentration at threshold level (1995 version) expressed in molar fraction, $\mathrm{n}$ stands for standardized power function exponent according to Devos et al. [25], MR stands for molar refraction and $\mathrm{Vw}$ for Van der Waals molar volume (the two later according to the Chemaxon calculator-plugins [15]), and at last PSA2 stands for the new proposed expression of the polar surface area, as defined in the Methods section. The numbers situated below the three independent variables of Equation (1) stand for the par- 
tial $\mathrm{F}$ ratios corresponding to each term.

The global characteristics of the regression are:

$\mathrm{r}=0.77, \mathrm{~N}=186, \mathrm{~F}=89$, without outliers

Equation (1) means that the stepwise regression analysis:

- has confirmed the selection of $1 / \mathrm{n}$,

- has selected one of the terms more or less related to the molecular size, with a preference for molar refraction,

- has selected an expression of the polarity expressed by the ratio PSA2/Vw (preference of the new expression of PSA and preference of $\mathrm{Vw}$ over $\mathrm{Sw}$, among others),

- has not selected the proposed induction/ polarizability expression $(\delta)$,

- has not selected ClogP (partition coefficient octanolwater), very popular in pharmacology.

In order to simplify the Equation (1) handling, we propose to name vertolf (as VERtebrates Olfactory Filter), the sum of its second and third terms:

$$
\text { vertolf }=\frac{7.406 \times \mathrm{MR}}{100}+3.604 \times \frac{\mathrm{PSA} 2}{\mathrm{Vw}}
$$

Because of the definition of p.ol, all the terms of Equation (1) can be multiplied by $(-1)$ if the dependent variable is preferred as the log of threshold (also expressed in molar fraction):

$$
\log \text { threshold }=-\frac{0.8177}{n}-\text { vertolf }-2.3
$$

Equation (3) can be compared to that obtained by Patte et al. [7] for the honey-bees:

$$
\log C_{0}=-\frac{\log R_{C}-\log R_{0}}{n}+\log \mathrm{SVP}-2.4
$$

A comparison between Equations (3) and (4) clearly shows an almost identical value of the constant, corresponding to the convergence point abscissa in the bottom of Figure 1 (i.e. approximately the $\log$ value of 1/200 the supposed maximal concentration at the level of recaptors).

In addition, [- vertolf] expression takes the place of log SVP, and we can interpret the numerator of the first term in Equation (3) as follows:

$$
\log R_{C}-\log R_{0}=0.8177
$$

or:

$$
\frac{R_{C}}{R_{0}}=6.57
$$

\subsection{Nature of the Convergence Point Ordinate}

The challenge is, as we saw, to check if the ratio $R_{C} / R_{0}$ equal to 6.57 corresponds or not to an easily noticeable level, not too faint, not too strong.

The oldest attempt of a standardized scale of OLPI is credited to Allison and Katz in 1919 [28]. According to this method, named category scaling, when subjects are asked to classify odor intensities in, for example, one of these categories: odorless, very weak, weak, medium, strong, very strong, by attributing a such value as $0,1,2$, 3,4 or 5 to each of these categories, a linearity between these numbers and the logarithm of the concentration is observed. In some ways, these categories are directly proportional to a logarithmic expression of the true OLPI.

In a more accurate method, named olfactory matching, odorous stimuli under study may be compared with a series of calibrated concentrations of a reference odor, generally 1-butanol. Initiated in 1971 by Dravnieks [29], this procedure has been adopted as a standard method by the ASTM [30] and by AFNOR [31]. In the latter standard, correspondences between the two scales are indicated.

We have displayed in Figure 3 the corresponding values between the suprathreshold categories and the 1-butanol concentration equivalences according to the AFNOR standard X 43-103 [31]. Figure 3 also includes the corresponding standardized values of the OLPI according to the equation proposed by Devos et al. [27]:

$$
\mathrm{OLPI}_{2002}=\sqrt{2 C}
$$

\begin{tabular}{|c|c|c|c|c|}
\hline Category & $\begin{array}{l}\text { log ppm } \\
\text { 1-butanol }\end{array}$ & $\begin{array}{c}\text { ppm } \\
\text { 1-butanol }\end{array}$ & $\begin{array}{l}\text { OLPI } \\
2002\end{array}$ & Particular cases \\
\hline Very strong $\uparrow$ & $\begin{array}{l}3.0 \\
2.5\end{array}$ & $\begin{array}{l}1000 \\
316\end{array}$ & $\begin{array}{l}44.70 \\
25.10\end{array}$ & \\
\hline Strong & $\begin{array}{l}2.0 \\
1.5\end{array}$ & $\begin{array}{r}100 \\
31.6\end{array}$ & $\begin{array}{r}14.10 \\
7.90\end{array}$ & \\
\hline Easily noticeable & $\begin{array}{r}1.22 \\
1.0 \\
0.5\end{array}$ & $\begin{array}{l}16.7 \\
10.0 \\
3.16\end{array}$ & $\begin{array}{l}5.78 \\
4.50 \\
2.50\end{array}$ & $\begin{array}{c}\text { Convergence } \\
\text { point ordinate } \\
\text { (present study) }\end{array}$ \\
\hline Faint & $\begin{array}{r}0.0 \\
-0.41 \\
-0.5 \\
\end{array}$ & $\begin{array}{r}1.00 \\
0.39 \\
0.316 \\
\end{array}$ & $\begin{array}{l}1.40 \\
0.88 \\
0.80\end{array}$ & $\leftarrow \begin{array}{l}\text { Std. threshold } \\
(1995)^{25}\end{array}$ \\
\hline Very faint & $\begin{array}{l}-1.0 \\
-1.5\end{array}$ & $\begin{array}{l}0.100 \\
0.032\end{array}$ & $\begin{array}{l}0.50 \\
0.25\end{array}$ & \\
\hline
\end{tabular}

in which $C$ stands for a given odorous intensity expressed in terms of ppm of 1-butanol. From this equation, 10 units of OLPI2002 correspond to $50 \mathrm{ppm}$ of 1-butanol.

Equation (6) applied to the threshold value of 1-butanol according to Devos et al. [25] (0.39 ppm vol) gives

Figure 3. Some corresponding values of three expressions of suprathreshold olfactory perceived intensities (OLPI). See text. 
an $\mathrm{OLPI}_{2002}$ value of 0.88 , and therefore the $\mathrm{R}_{\mathrm{C}}$ value of Equation $(5)$ becomes equal to $5.78(0.88 \times 6.57)$.

It clearly appears in Figure $\mathbf{3}$ that the convergence point ordinate takes the upper place in the easily noticeable category and it should therefore be suitable to attribute it the label of comfort point. It should however be specified that this assertion implies the procedures taken as a benchmark in the two compilations of Devos et al. and in the AFNOR standard as well, i.e. the natural snifffing. Using other procedures, as for example a relatively low flow rate of stimulation (e.g. $160 \mathrm{ml} / \mathrm{min}$, as recommended by ASTM E544-99), leads to a reaching easily noticeable category for higher concentrations of 1-butanol (around 87 ppm according to Dravnieks [32]). It can be seen in Figure 3 that this concentration using natural sniffing corresponds to a strong category.

\section{Discussion}

\subsection{The Model of Comfort OLPI Here Proposed}

We have observed some satisfactory results in the previous section, when compared to those previously obtained in the honey-bee:

- the abscissas of the convergence point in the bottom of Figure 1 are very similar in both cases [values of constants in Equations (3) an (4)]

- the ordinate of the convergence point in the same bottom of Figure 1 corresponds in both cases to an OLPI not too strong, not too faint, optimal for odorant recognition by the honey-bees (Introduction), and characterized as easily noticeable from human responses (Figure 3).

By contrast, the statistical tests obtained between experimental and predicted human olfactory thresholds using Equation (1) are appreciably lower than in the honey-bee experimentation $(\mathrm{r}=0.77$ instead of $0.97, \mathrm{~F}=$ 89 instead of 404). One argument seems to indicate that this observation is rather due to a lack of accuracy of the biological data than the weakness of the model:

We have compared our above results using Equation (1) with the predicted human olfactory thresholds in the three most recent publications on this QSAR topic, even not at all involved in the comfort concept here developed [33-35]. From the comparison of statistical tests, the F ratio values are moderate in all cases, and incidentally the best one is observed with the present study, as summarized in Table 1. As a reminder, $F$ ratio value can be obtained from the correlation coefficient $r$, the number of observations $\mathrm{N}$ (here the number of odorants) and the number of independent variables, according to Abdi [36]:

$$
F=\frac{r^{2}}{1-r^{2}} \times \frac{N-N b \text { IndepVar }-1}{\text { NbIndepVar }}
$$

Table 1. Comparative statistical tests SEE, $r$ and $F$ observed for six QSAR models in three recent publications [33-35] and in the present study.

\begin{tabular}{cccccccc}
\hline \multicolumn{1}{c}{ Authors } & Indep. Var. & N & SEE & $\mathbf{r}$ & $\mathbf{F}$ \\
\hline \multicolumn{7}{c}{ Physiol } & Chem \\
\hline $\begin{array}{c}\text { Abraham } \text { et al. } \\
(2002)\end{array}$ & Equation (6) & 0 & 5 & 50 & 0.58 & 0.88 & 29 \\
& Equation (9) & 0 & 7 & 60 & 0.60 & 0.92 & 44 \\
$\begin{array}{c}\text { Abraham et al. } \\
(2012)\end{array}$ & Equation (5) & 0 & 9 & 193 & 0.83 & 0.86 & 60 \\
$\begin{array}{c}\text { Zarzo (2012) } \\
\text { present work }\end{array}$ & Equation (6) & 6 & 9 & 353 & 0.82 & 0.87 & 71 \\
\hline
\end{tabular}

Obviously, the quality of the prediction differs according to the considered statistical test, $\mathrm{r}$ or $F$. The statisticians appear to favor the second criterion, particularly when the number of independent variables increases in large proportion relatively to the number of observations (here the odorants under study).

One of the possible applications of the model of OLPI comfort proposed here is the prediction of power law exponents from experimental data sets of olfactory thresholds, which are known for much more odorants. That could be useful, for example, in the study of synergy and inhibition of olfactory mixtures, where it has be shown for a long time the role played by the power law exponents $[37,38]$. More generally speaking, the suprathreshold OLPI using ASTM or AFNOR standard procedures should be applied more frequently in place or in complement of thresholds measurements.

\subsection{The Polar Surface Area}

The finding that the ratio of the polar surface area over the Van der Waals molecular volume (PSA/Vw) has been selected by the MLRA processing in the present study may be related to the involving of this expression in gas-liquid chromatographic phenomena, where it estimates well the polarity of stationary phases (Laffort [3] ). At this stage, only the classical polar surface area, i.e. PSA1, has been tested in chromatographic phenomena.

The PSA2 version, apparently more rational on a theoretical point of view than the PSA1 and PSA3 versions, has been always, in the present study, preferred by the MLRA processing to the two other versions.

\section{Conclusion}

The cross validation on the comfort OLPI from honeybee and human responses in one hand, and on the expression PSA/Vw from chromatographic phenomena and 
the present psychophysical study in the other hand, provides strong arguments in favor of a robustness of the whole.

\section{Supporting Information}

Supporting information associated with this article (numerical data of physicochemical and olfactory properties for 186 odorants) is freely available on request to the author: <paul.laffort@u-bourgogne.fr>.

\section{Acknowledgements}

The author warmly acknowledges David Laffort for his editing assistance, and also sincerely thanks the ChemAxon Company for its freely interactive calculator. He is strongly indebted to the co-authors involved in the previous studies making possible the present synthesis, more particularly: Gérard Arnold, Isabelle Canard, Michel Devos, Andrew Dravnieks, Michel Etcheto, Pierre Héricourt, Pierrette Marfaing, François Patte, Yves Pichon, Jacques Rouault, and Leo Van Gemert. Thank you so much to all!

\section{REFERENCES}

[1] K. Palm, K. Luthman, A. L. Ungell, G. Strandlund and P. Artursson, "Correlation of Drug Absorption with Molecule Surface Properties," Journal of Pharmaceutical Sciences, Vol. 85, No. 1, 1996, pp. 32-39. http://dx.doi.org/10.1021/js950285r

[2] K. Palm, P. Stenberg, K. Luthman and P. Artursson, "Polar Molecular Surface Properties Predict the Intestinal Absorption of Drugs in Humans," Pharmaceutical Research, Vol. 14, No. 5, 1997, pp. 568-571. http://dx.doi.org/10.1023/A:1012188625088

[3] P. Laffort, "Solvation Parameters. Part 5: Physicochemical Interpretation of Experimental Solvent Values for Stationary Phases of Gas-Liquid Chromatography," Journal of Chromatography A, Vol. 1218, No. 26, 2011, pp. 4025-4033. http://dx.doi.org/10.1016/j.chroma.2011.04.068

[4] P. Ertl, "Polar Surface Area," In: R. Mannhold, Ed., Molecular Drug Properties, Wiley-VCH, Heidelberg, 2007, pp. 111-126. http://dx.doi.org/10.1002/9783527621286.ch5

[5] M. Muehlbacher, G. M. Spitzer, K. R. Liedl and J. Kornhuber, "Qualitative Prediction of Blood-Brain Barrier Permeability on a Large and Refined Dataset," Journal of Computer-Aided Molecular Design, Vol. 25, No. 12, 2011, pp. 1095-1106. http://dx.doi.org/10.1007/s10822-011-9478-1

[6] P. Laffort and A. Dravnieks, "An Approach to a PhysicoChemical Model of Olfactory Stimulation in Vertebrates by Single Compounds," Journal of Theoretical Biology, Vol. 38, No. 2, 1973, pp. 335-345.

http://dx.doi.org/10.1016/0022-5193(73)90178-1

[7] F. Patte, M. Etcheto and P. Marfaing, "Laffort Electroan- tennogram Stimulus-Response Curves for 59 Odourants in the Honey-Bee Apis mellifica," Journal of Insect Physiology, Vol. 35, No. 9, 1989, pp. 667-675. http://dx.doi.org/10.1016/0022-1910(89)90086-3

[8] P. Marfaing, J. Rouault and P. Laffort, "Effect of the Concentration and Nature of Olfactory Stimuli on the Proboscis Extension of Conditioned Honey Bees Apis mellifica Ligustica," Journal of Insect Physiology, Vol. 35, No. 12, 1989, pp. 949-955.

http://dx.doi.org/10.1016/0022-1910(89)90018-8

[9] I. Canard, "Influence de L'intensité du Stimulus et de L'ionisation de L'atmosphère sur L'apprentissage Olfactif de l'Abeille Apis mellifica Ligustica," DEA of Behavior Biology, University of Paris, 1990.

[10] I. Canard, M. Devos and P. Laffort, "Influence de L'intensité du Stimulus et de L'ionisation de L'atmosphère sur L'apprentissage Olfactif de L'Abeille," 10th Meeting on Insect Physiology, Toulouse, 19-21 September 1990.

[11] P. Laffort and F. Patte, "Derivation of Power Law Exponents from Olfactory Thresholds for Pure Substances," Annals of the New York Academy of Sciences, Vol. 510, 1987, pp. 436-439.

http://dx.doi.org/10.1111/j.1749-6632.1987.tb43582.x

[12] F. Chauvin, "Amélioration de la Définition et de la Détermination des Paramètres de Solubilité. Application à L'olfaction," Ph.D. Thesis, University of Bourgogne, Dijon, 1998.

[13] P. Laffort, “Apprentissage Olfactif chez L'Abeille: Quelques Résultats Susceptibles D'extension à l'Homme," In: G. Bertrand, Ed., Le Goût, Network CLUSE, Third CrossBorder Symposium, 12-13 September 1996, University of Bourgogne, Dijon, 1998, pp. 579-584.

[14] P. Laffort, "The Concept of Comfort Concentration in Olfaction from Honey Bee Experimentation. Similarities and Differences with Human Olfaction," Monell and TOSTQ Sweetness Conference Workshop, 23-24 April 2001, Monell Chemical Senses Centre, Philadelphia.

[15] ChemAxon Ltd., 2012. http://www.chemaxon.com/products/calculator-plugins

[16] P. Laffort and P. Héricourt, "Solvation Parameters of Solutes: An Attempt of Improvements in Characterization and Determination," The Open Applied Informatics Journal, Vol. 2, 2008, pp. 22-31. http://dx.doi.org/10.2174/1874136300802010022

[17] P. Ertl, B. Rohde and P. Selzer, "Fast Calculation of Molecular Polar Surface Area as a Sum of Fragment-Based Contributions and Its Application to the Prediction of Drug Transport Properties," Journal of Medical Chemistry, Vol. 43, No. 20, 2000, pp. 3714-3717. http://dx.doi.org/10.1021/jm000942e

[18] S. Winiwarter, N. M. Bonham, F. Ax, A. Hallberg, H. Lennernäs and A. Karlén, "Correlation of Human Jejunal Permeability (in Vivo) of Drugs with Experimentally and Theoretically Derived Parameters. A Multivariate Data Analysis Approach," Journal of Medical Chemistry, Vol. 41, No. 25, 1998, pp. 4939-4949. http://dx.doi.org/10.1021/jm9810102

[19] G. N. Lewis, "The Atom and the Molecule," Journal of the American Chemical Society, Vol. 38, No. 4, 1916, pp. 
762-785. http://dx.doi.org/10.1021/ja02261a002

[20] M. H. Abraham, "Scales of Solute Hydrogen-Bonding: Their Construction and Application to Physicochemical and Biochemical Processes," Chemical Society Reviews, Vol. 22, No. 2, 1993, pp. 73-83. http://dx.doi.org/10.1039/cs9932200073

[21] A. M. Zissimos, M. H. Abraham, A. Klamt, F. Eckert and J. Wood, "A Comparison between the Two General Sets of Linear Energy Descriptors of Abraham and Klamt," Journal of Chemical Information and Computer Sciences, Vol. 42, No. 6, 2002, pp. 1320-1331. http://dx.doi.org/10.1021/ci025530o

[22] L. J. Van Gemert, "Compilations of Odour Threshold Values in Air, Water and Other Media," Oliemans, Punter \& Partners BV, Zeist, 2011.

[23] P. Laffort, "Essai de Standardisation des Seuils Olfactifs Humains pour 192 Corps Purs,"Archives des Sciences Physiologiques, Vol. 17, 1963, pp. 75-105.

[24] M. Devos, F. Patte, J. Rouault, P. Laffort and L. J. Van Gemert, "Standardized Human Olfactory Thresholds in Air," IRL Press, Oxford, 1990.

[25] M. Devos, J. Rouault, P. Laffort and L. J. Van Gemert, "Standardized Human Olfactory Thresholds in Air," Unpublished Updating of the 1990 Version, 1995.

[26] W. S. Cain, "Odor Intensity Differences in the Exponent of the Psychophysical Function," Perception \& Psychophysics, Vol. 6, No. 6, 1969, pp. 349-354. http://dx.doi.org/10.3758/BF03212789

[27] M. Devos, J. Rouault and P. Laffort, "Standardized Olfactory Power Law Exponents in Man," Editions Universitaires de Dijon, Dijon, 2002.

[28] V. C. Allison and S. H. Katz, "An Investigation of Stenches and Odors for Industrial Purposes," Journal of Industrial \& Engineering Chemistry, Vol. 11, No. 4, 1919, pp. 336-339. http://dx.doi.org/10.1021/ie50112a024

[29] A. Dravnieks, "Fundamentals of Odor Perception. Their Applicability to Air Pollution Control Programs," NCASI Technical Bulletin, Vol. 54, 1971, pp. 1-27.

[30] ASTM Historical Standard: ASTM E544-99, "Standard Practices for Referencing Suprathreshold Odor Intensity,"
American Society for Testing and Materials, Philadelphia, 2004.

[31] AFNOR, “Air Quality, Olfactometric Measurements, Measurement of the Odour of Gaseous Effluent, Supra-Threshold Methods," French Standard AFNOR NF X 43-103, Paris, 1996.

[32] A. Dravnieks, "Correlation of Odor Intensities and Vapor Pressures with Structural Properties of Odorants," In: R. A. Scanlon, Ed., Flavor Quality, Objective Measurement, ACS Symposium Series, Vol. 51, American Chemical Society, Washington DC, 1977, pp. 11-28. http://dx.doi.org/10.1021/bk-1977-0051.ch002

[33] M. H. Abraham, J. M. R Gola, J. E. Cometto-Muñiz and W. S. Cain, "A Model for Odour Thresholds," Chemical Senses, Vol. 27, No. 3, 2002, pp. 95-104. http://dx.doi.org/10.1093/chemse/27.2.95

[34] M. H. Abraham, R. Sánchez-Moreno, J. E. Cometto-Muñiz and W. S. Cain, "An Algorithm for 353 Odor Detection Thresholds in Humans," Chemical Senses, Vol. 37, No. 3, 2012, pp. 207-218. http://dx.doi.org/10.1093/chemse/bjr094

[35] M. Zarzo, "Effect of Functional Group and Carbon Chain Length on the Odor Detection Threshold of Aliphatic Compounds," Sensors, Vol. 12, No. 4, 2012, pp. 41054112. http://dx.doi.org/10.3390/s120404105

[36] H. Abdi, "Multiple Correlation Coefficient," In: N. J. Salkind, Ed., Encyclopedia of Measurement and Statistics, Sage, Thousand Oaks, 2007, pp. 648-651. http://dx.doi.org/10.4135/9781412952644.n91

[37] P. Laffort, M. Etcheto, F. Patte and P Marfaing, "Implications of Power Law Exponent in Synergy and Inhibition of Olfactory Mixtures," Chemical Senses, Vol. 14, No. 1, 1989, pp. 11-23. http://dx.doi.org/10.1093/chemse/14.1.11

[38] P. Laffort, "Inhibitory Process of the Olfactory Perception," French Patent No 9308079, CNRS, 1993. PCT Extension in Europe, USA and Canada in 1994, Ratified in Europe in 1995 and First Semester 1996 (16 Contracting Countries of the Agreement on the European Patent) Come into the Public Domain in July 1996. 